\title{
The Chemistry of Dinuclear Analogs of the Anticancer Drug Cisplatin. A DFT/CDM Study
}

\author{
Dirk V. Deubel*
}

ETH Zurich, USI Campus, Computational Science, D-CHAB, 6900 Lugano, Switzerland metals-in-medicine@phys.chem.ethz.ch,http://www.staff.uni-marburg.de/ deubel

\section{Supporting Information}

Table S-1. Comparison of calculated and experimental activation free energies $\left(\Delta \mathrm{G}_{\mathrm{a}}\right.$; in $\mathrm{kcal} / \mathrm{mol})$ and reaction free energies $\left(\Delta \mathrm{G}_{\mathrm{r}}\right.$; in $\left.\mathrm{kcal} / \mathrm{mol}\right)$ for the hydrolysis of Pt-Cl bonds anticancer complexes, and absolute $\mathrm{pK}_{\mathrm{a}}$ values of the aqua complexes. ${ }^{\mathrm{a}}$

\begin{tabular}{|c|c|c|c|c|}
\hline Metal complex & & calc. & exp. & exp. ref. \\
\hline cis- $\left[\mathrm{Pt}\left(\mathrm{NH}_{3}\right)_{2} \mathrm{Cl}_{2}\right](\mathbf{A})$ & $\Delta \mathrm{G}_{\mathrm{a}}$ & 24.9 & $23.8 ; 24.1$ & $\mathrm{c} ; \mathrm{d}$ \\
\hline$c i s-\left[\mathrm{Pt}\left(\mathrm{NH}_{3}\right)_{2} \mathrm{Cl}_{2}\right](\mathbf{A})$ & $\Delta \mathrm{G}_{\mathrm{r}}$ & 0.1 & $4.2 ; 3.6$ & $c ; d$ \\
\hline$c i s-\left[\mathrm{Pt}\left(\mathrm{NH}_{3}\right)_{2}\left(\mathrm{OH}_{2}\right) \mathrm{Cl}\right]^{+}(\mathbf{B})$ & $\Delta \mathrm{G}_{\mathrm{a}}$ & 25.3 & 23.3 & $\mathrm{~d}$ \\
\hline$[\mathrm{Ru}(\mathrm{Ar})(\mathrm{en}) \mathrm{Cl}]^{+b}$ & $\Delta \mathrm{G}_{\mathrm{a}}$ & 20.7 & 21.4 & $\mathrm{~b}$ \\
\hline$[\mathrm{Ru}(\mathrm{Ar})(\mathrm{en}) \mathrm{Cl}]^{+b}$ & $\Delta \mathrm{G}_{\mathrm{r}}$ & 1.1 & 3.2 & $\mathrm{~b}$ \\
\hline$c i s-\left[\mathrm{Pt}\left(\mathrm{NH}_{3}\right)_{2}\left(\mathrm{OH}_{2}\right) \mathrm{Cl}\right]^{+}\left(\mathbf{B}, \mathrm{pK}_{\mathrm{a} 1}\right)$ & $\mathrm{pK}$ & 7.8 & 6.41 & $\mathrm{e}$ \\
\hline$c i s-\left[\mathrm{Pt}\left(\mathrm{NH}_{3}\right)_{2}\left(\mathrm{OH}_{2}\right)_{2}\right]^{2+}\left(\mathbf{C}, \mathrm{pK}_{\mathrm{a} 2}\right)$ & $\mathrm{pK}_{\mathrm{a}}$ & 8.3 & 5.37 & $\mathrm{e}$ \\
\hline$c i s-\left[\mathrm{Pt}\left(\mathrm{NH}_{3}\right)_{2}\left(\mathrm{OH}_{2}\right)(\mathrm{OH})\right.$ & $\mathrm{pK}_{\mathrm{a}}$ & 9.5 & 7.21 & $\mathrm{e}$ \\
\hline$\left[\mathrm{Ru}(\mathrm{Ar})(\mathrm{en})\left(\mathrm{OH}_{2}\right)\right]^{2+}$ & $\mathrm{pK}_{\mathrm{a}}$ & 9.8 & 7.71 & b \\
\hline
\end{tabular}

${ }^{\mathrm{a}}$ A difference of $1 \mathrm{pK}_{\mathrm{a}}$ unit reflects a free energy difference of RTln $10=1.36 \mathrm{kcal} / \mathrm{mol}$. Table taken from ref. 19.

${ }^{\mathrm{b}}$ en $=1$,2-diaminoethane. $\mathrm{Ar}=\eta^{6}$-benzene (calc), $\eta^{6}$-biphenyl (exp). Wang, F.; Chen, H.; Parsons, S.; Oswald, I. D. H.; Davidson, J. E.; Sadler, P. J. Chem. Eur. J. 2003, 9, 5810.

${ }^{c}$ Coe, J. S. MTP Int. Rev. Sci.: Inorg. Chem., Ser. 2 1974, 45.

${ }^{\mathrm{d}}$ Perumareddi, J. R.; Adamson, A. W. J. Phys. Chem. 1978, 72, 414.

${ }^{\mathrm{e}}$ Berners-Price, S. J.; Frenkiel, T. A.; Frey, U.; Ranford J. D.; Sadler, P. J. Chem. Commun. 1992, 789.

Table S-2. Energy decomposition (at BP86, in vacuo, in $\mathrm{kcal} / \mathrm{mol}$ ) of [cis$\left.\mathrm{Pt}\left(\mathrm{NH}_{3}\right)_{2}(\mathrm{OH})_{2}\right]_{2}(\mathbf{8})$ in terms of two interacting monomers cis-[ $\left.\mathrm{Pt}\left(\mathrm{NH}_{3}\right)_{2}(\mathrm{OH})_{2}\right](\mathbf{F})\left(C_{s}\right.$ symmetry). Bold: Contributions from the irreducible representations $\Gamma_{I}$ to $\Delta E_{\text {orb }}$.

\begin{tabular}{lrr} 
Contribution & $\boldsymbol{\Gamma}_{\boldsymbol{i}}$ & value \\
\hline$\Delta E_{\text {str }}$ & & 12.2 \\
$\Delta E_{\text {Pauli }}$ & & 102.2 \\
$\Delta E_{\text {elst }}$ & & -97.3 \\
$\Delta E_{\text {orb }}$ & & -48.8 \\
$\boldsymbol{\Delta} E_{\text {orb }}\left(\Gamma_{i}\right)$ & $\boldsymbol{a}$ & $\mathbf{- 2 7 . 8}$ \\
& $\boldsymbol{a} ”$ & $\mathbf{- 2 1 . 0}$ \\
$\Delta E_{\text {total }}$ & & -31.7 \\
\hline
\end{tabular}


Table S-3. Free energies of the molecules and transition states shown in Figure 4 (in aqueous solution, relative to cisplatin, in $\mathrm{kcal} / \mathrm{mol}$ ).

\begin{tabular}{|c|c|c|c|}
\hline Molecule/ TS & value & Molecule/ TS & value \\
\hline (a) & & (e) & \\
\hline A & 0.0 & prod B Gua & -12.0 \\
\hline B & 0.1 & prod C Gua & -10.9 \\
\hline $\mathrm{C}$ & 1.9 & prod E Gua & 0.8 \\
\hline D & 10.8 & prod 5 Gua & 13.9 \\
\hline $\mathbf{E}$ & 13.1 & prod 7a Gua & 5.6 \\
\hline $\mathbf{F}$ & 26.0 & prod 7b Gua & 5.6 \\
\hline TS A B & 24.9 & prod B Met & -11.0 \\
\hline TS B C & 25.4 & prod C Met & -8.7 \\
\hline TS D E & 37.7 & prod $\mathbf{E}$ Met & 3.2 \\
\hline (b) & & prod 5 Met & 16.2 \\
\hline 1 & 1.1 & prod 7a Met & 6.1 \\
\hline 2 & 3.2 & prod 7b Met & 6.1 \\
\hline 3 & 4.7 & TS B Gua & 18.0 \\
\hline 4 & 12.4 & TS C Gua & 17.5 \\
\hline 5 & 7.4 & TS E Gua & 32.6 \\
\hline 6 & 28.5 & TS 5 Gua & 35.2 \\
\hline TS BD 1 & 30.9 & TS 7a Gua & 32.4 \\
\hline TS CD 2 & 31.4 & TS 7b Gua & 30.9 \\
\hline TS BE 2 & 32.3 & TS B Met & 21.0 \\
\hline TS CE 3 & 34.3 & TS C Met & 24.7 \\
\hline TS BF 4 & 41.5 & TS E Met & 34.4 \\
\hline TS ED 4 & 44.2 & TS 5 Met & 35.2 \\
\hline TS CF 5 & $\mathrm{a}$ & TS 7a Met & 31.4 \\
\hline TS EE 5 & 35.5 & TS 7b Met & 33.3 \\
\hline TS EF 6 & 55.0 & & \\
\hline (c) & & other molecules & \\
\hline TS 12 & $\mathrm{~b}$ & 8 & $2.3 \mathrm{c}$ \\
\hline TS 23 & $\mathrm{~b}$ & $9 a$ & $2.2 \mathrm{~d}$ \\
\hline TS 45 & 40.2 & $9 b$ & $4.2 \mathrm{~d}$ \\
\hline (d) & & & \\
\hline $7 a$ & 6.0 & & \\
\hline $7 b$ & 7.9 & & \\
\hline TS $47 \mathbf{a}$ & 38.7 & & \\
\hline TS 4 7b & 35.8 & & \\
\hline TS 5 7a & 22.5 & & \\
\hline TS 5 7b & 26.7 & & \\
\hline
\end{tabular}

${ }^{a}$ TS optimization yields TS EE5

${ }^{\mathrm{b}}$ Not calculated; the barrier is estimated to be similar to those for the first, second, and third hydrolysis.

${ }^{\mathrm{c}}$ Free energy given relative to 2 molecules of $\mathbf{F}$.

${ }^{\mathrm{d}}$ Free energy given relative to 1.5 molecules of $7 \mathbf{a}$. 
Figure S-1. Separated parts (a) - (e) of Figure 4.

$\mathrm{kcal} / \mathrm{mol}$

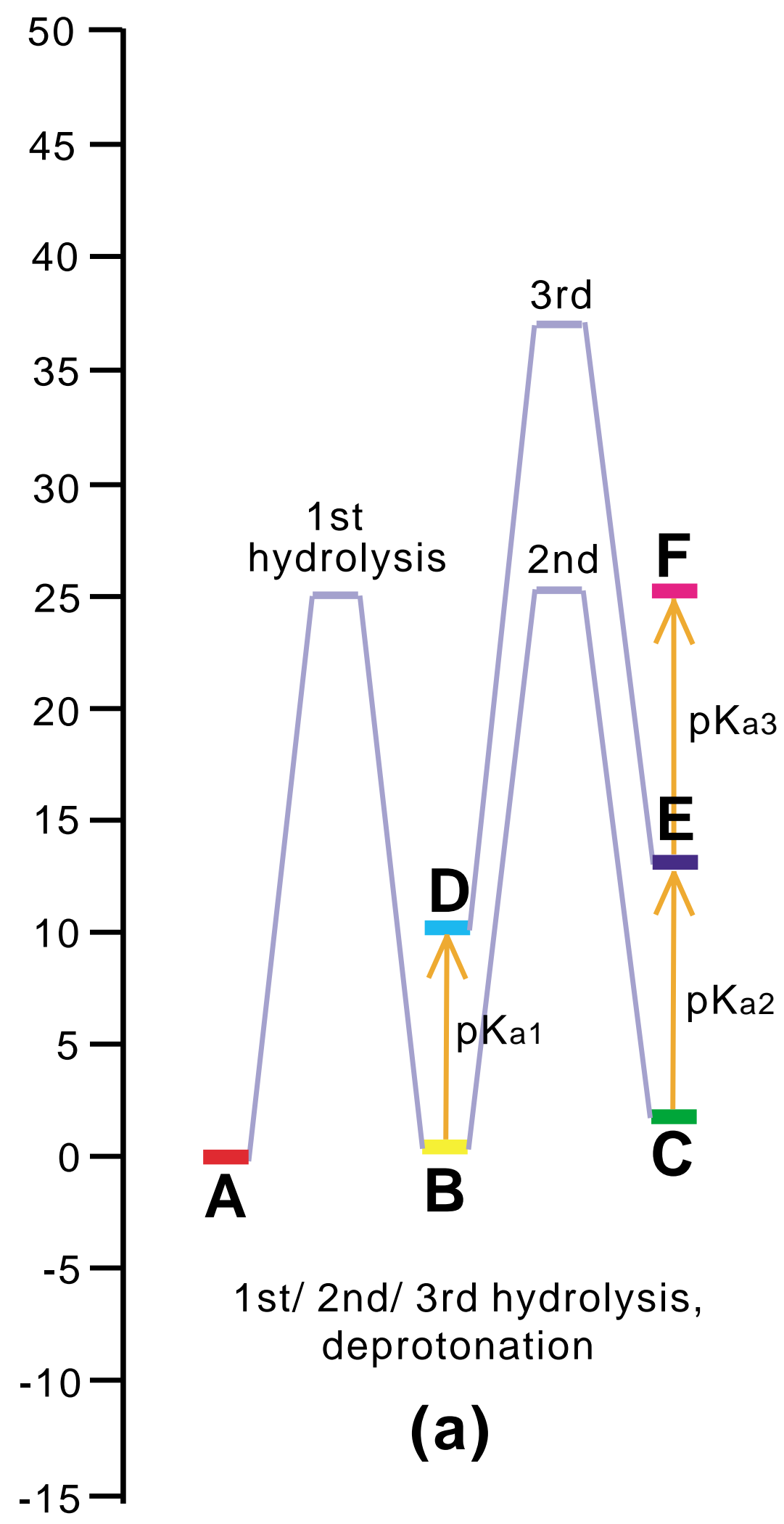




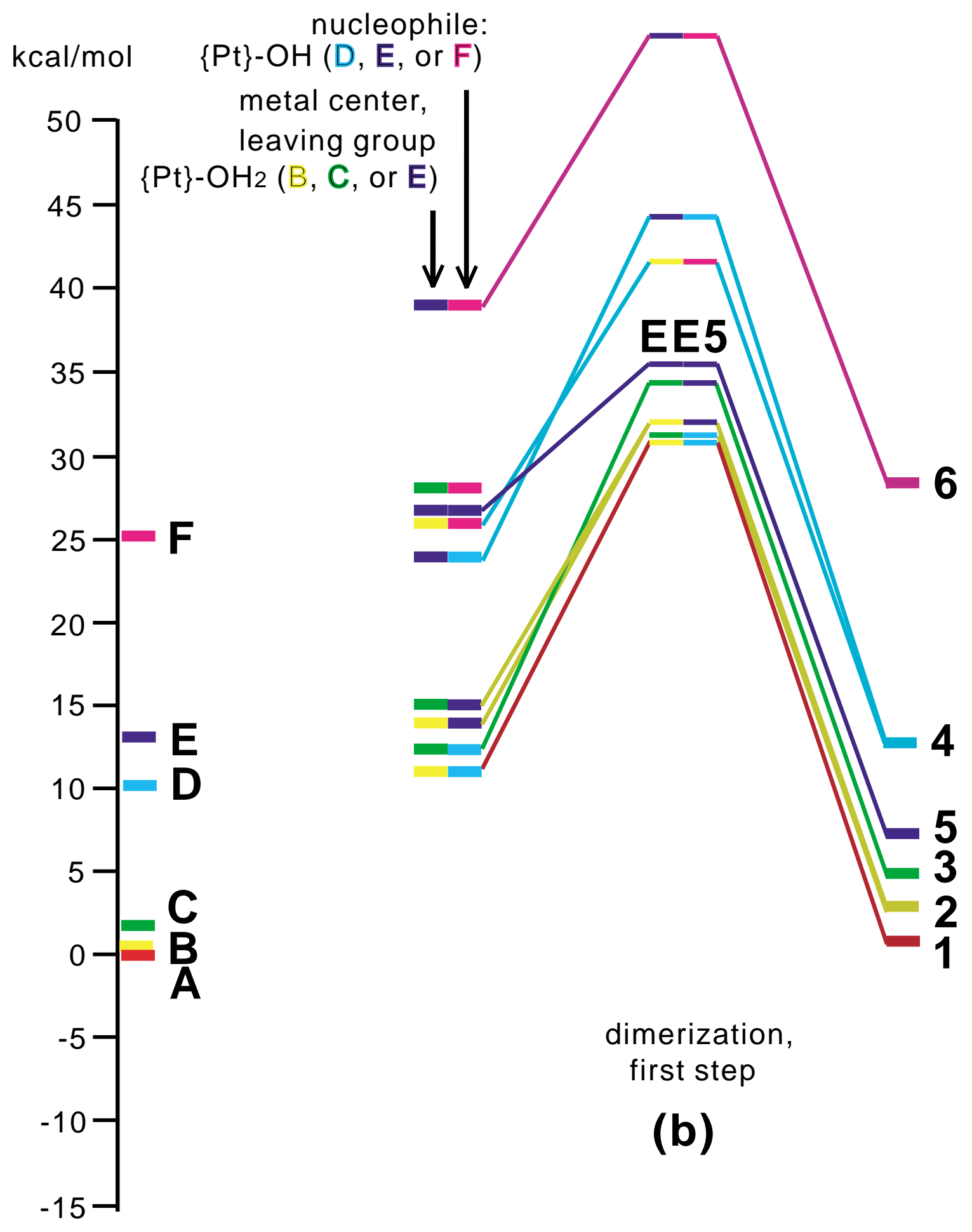


$\mathrm{kcal} / \mathrm{mol}$

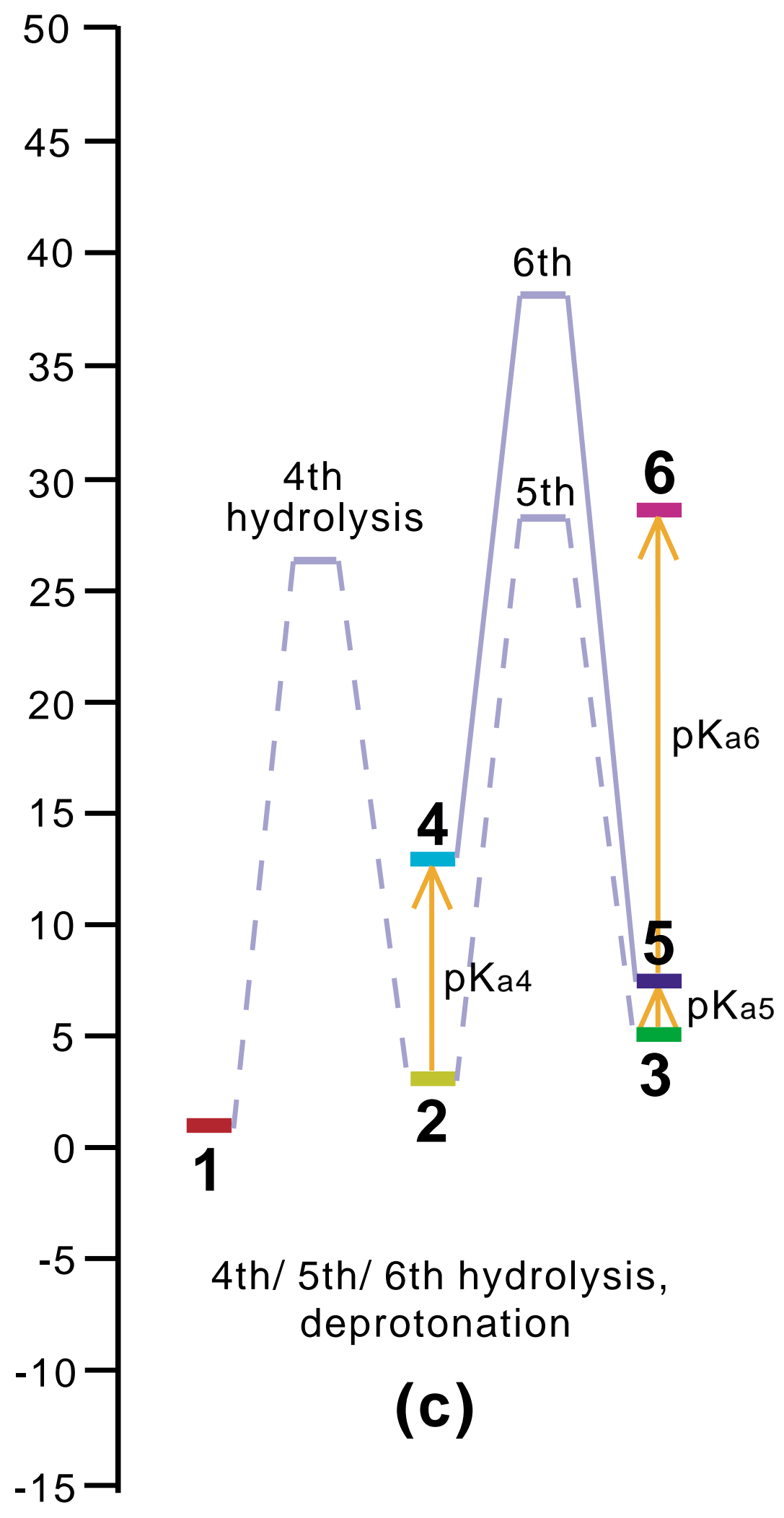


$\mathrm{kcal} / \mathrm{mol}$

50

45

$40-$

35

$30-$

25

20

5

10

$10-$

$5-$

$5-$ 


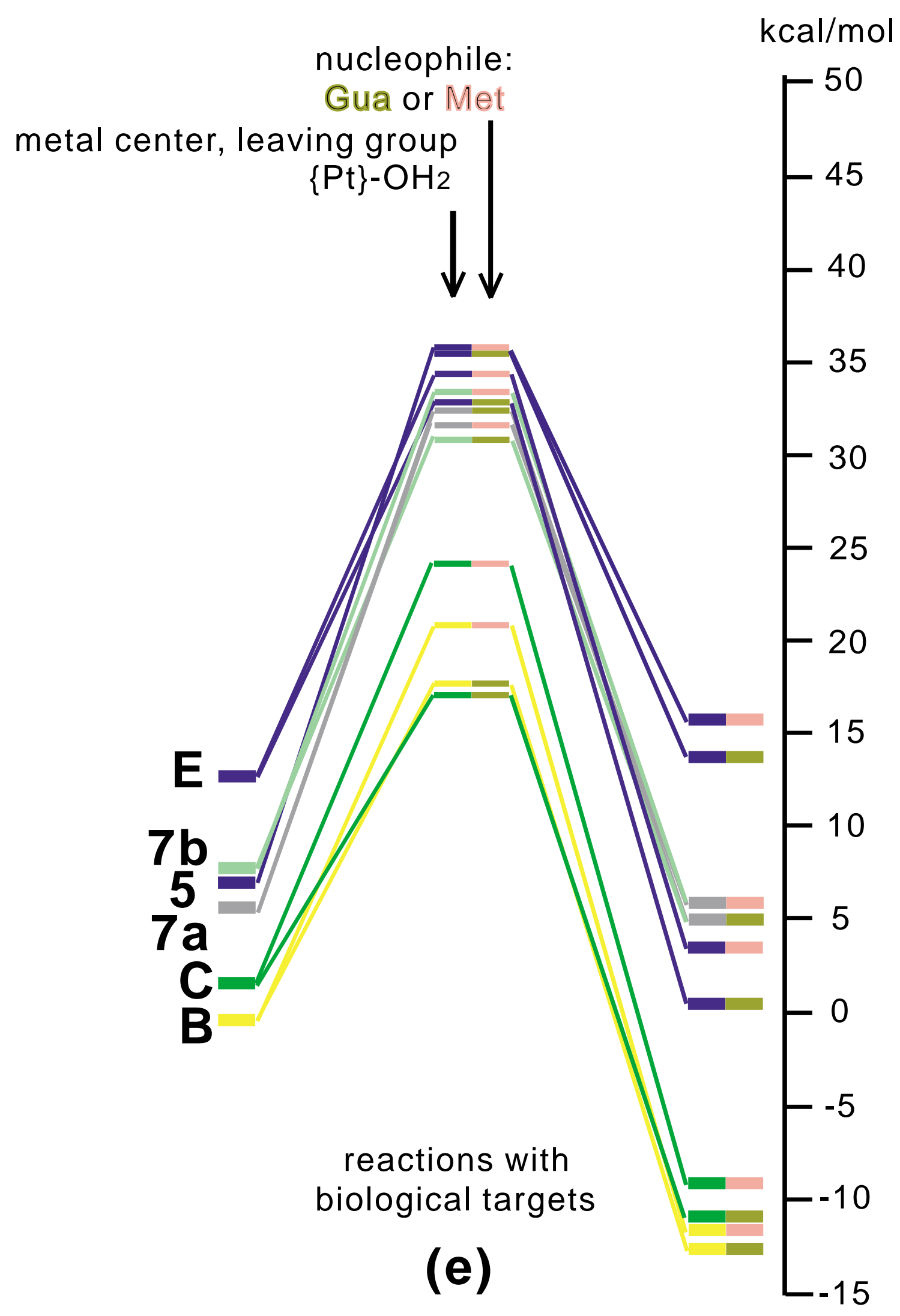


Figure S-2. Calculated structures. Distances in $\AA$.
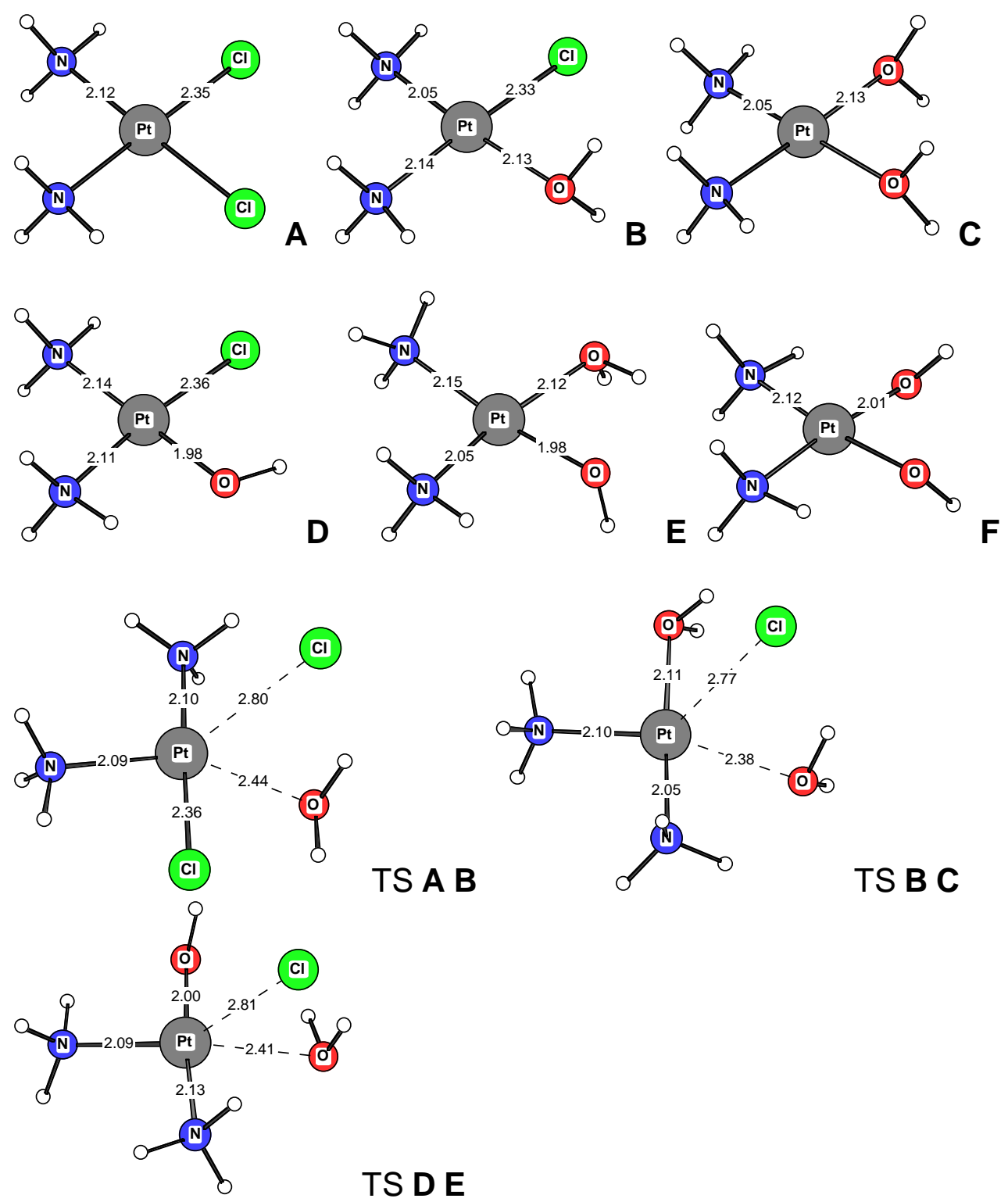

TS DE 

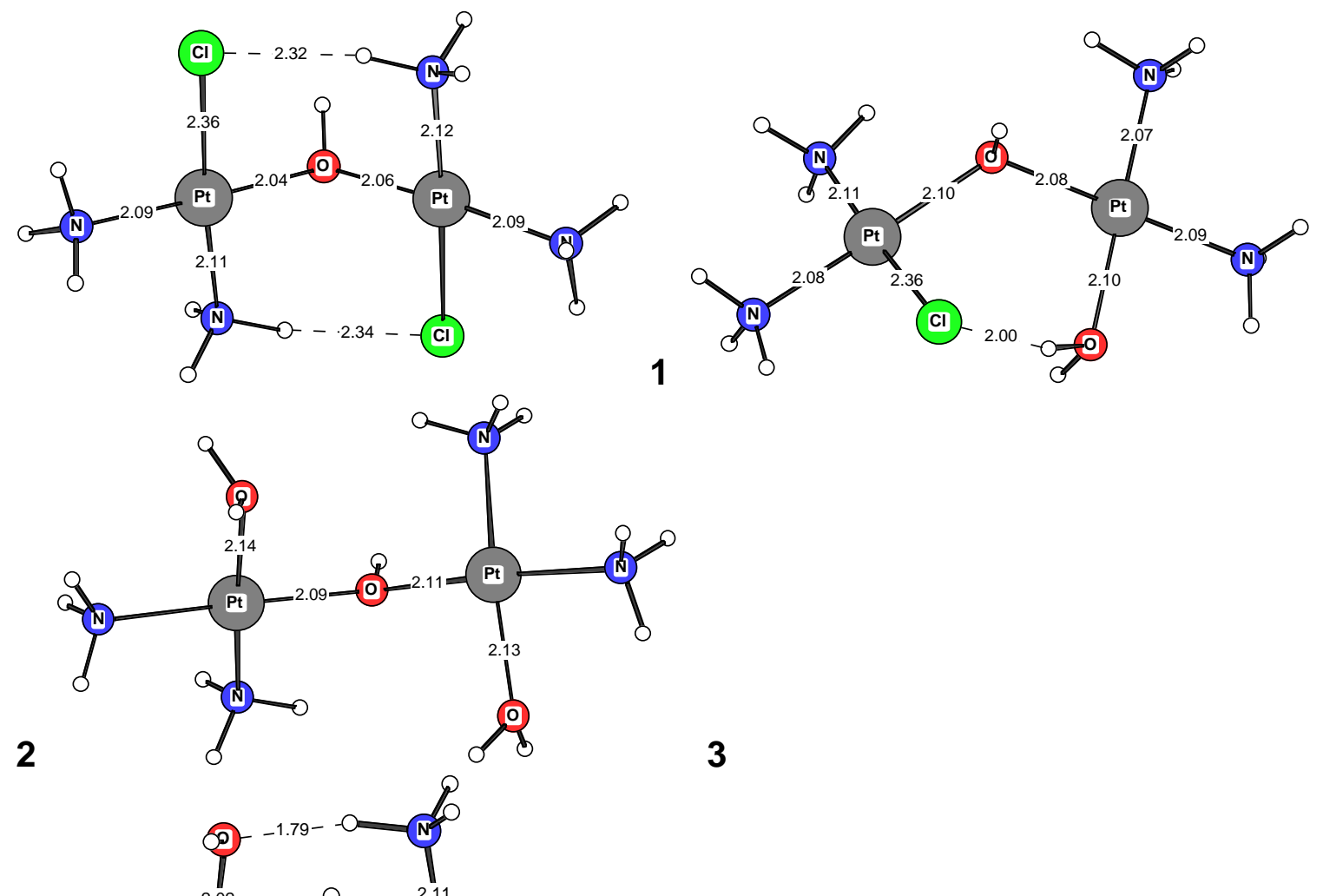

3
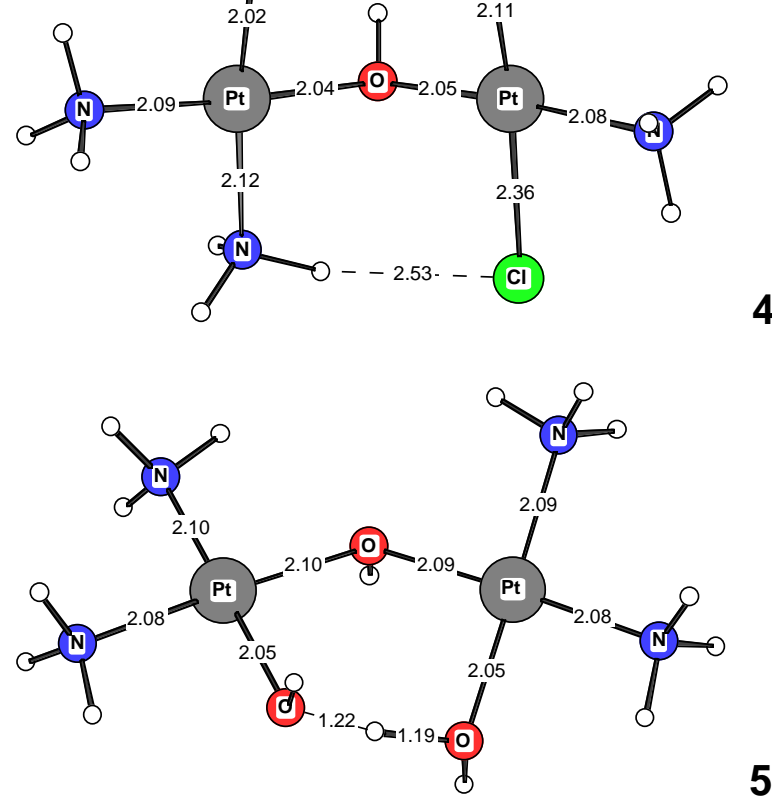

4

5 


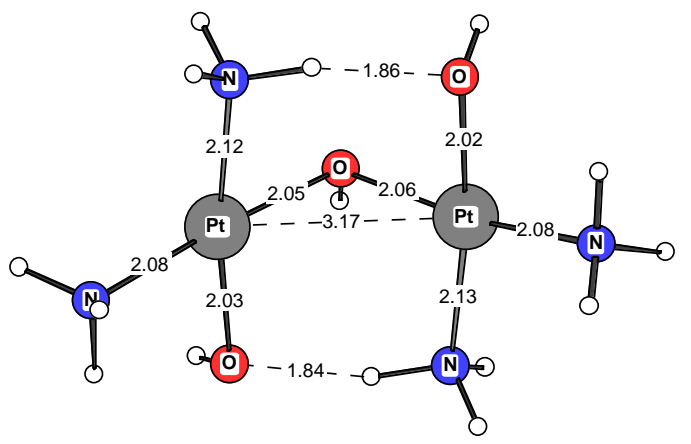

6

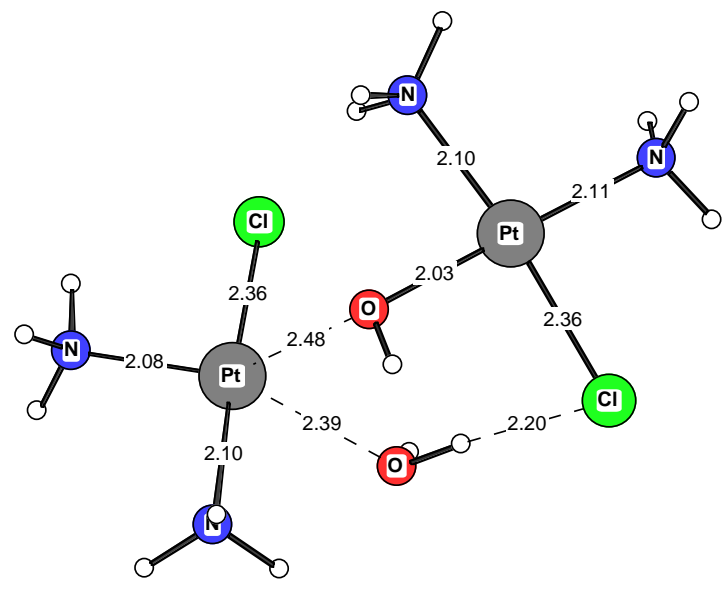

TS BD 1

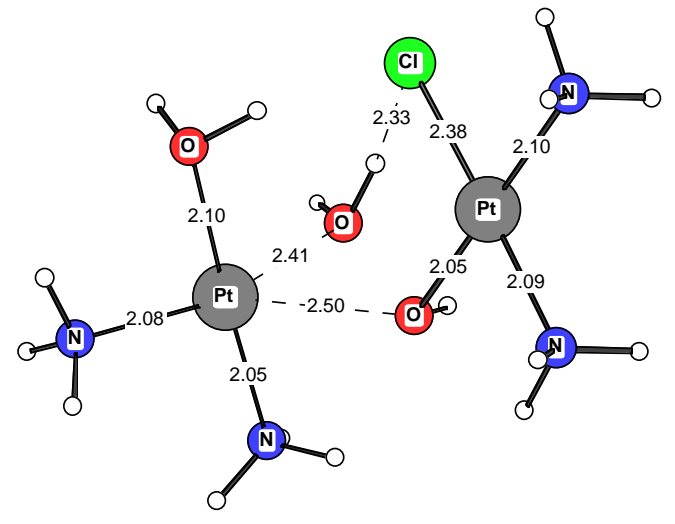

TS CD 2

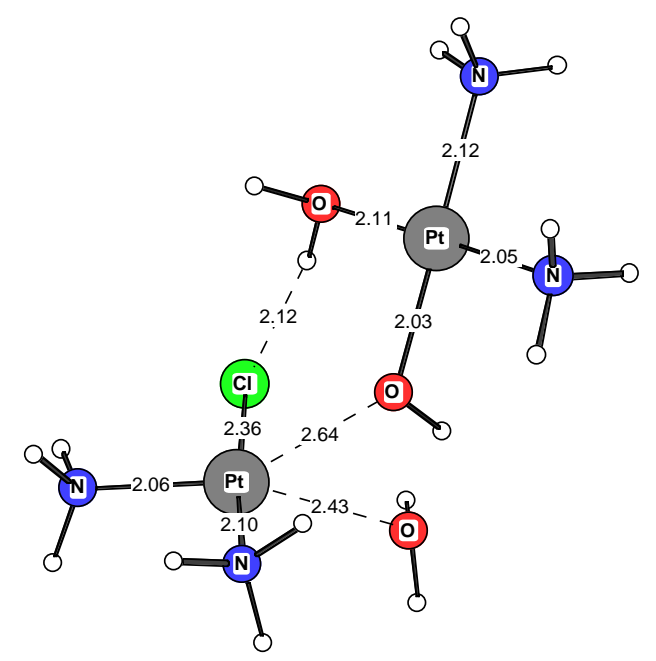

TS BE 2 


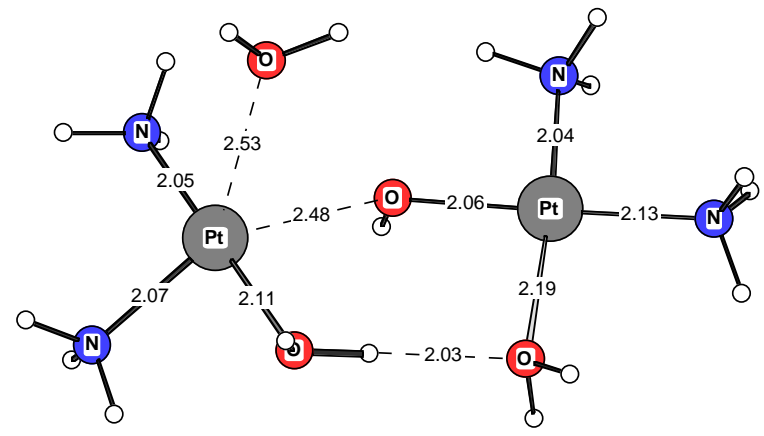

TS CE 3

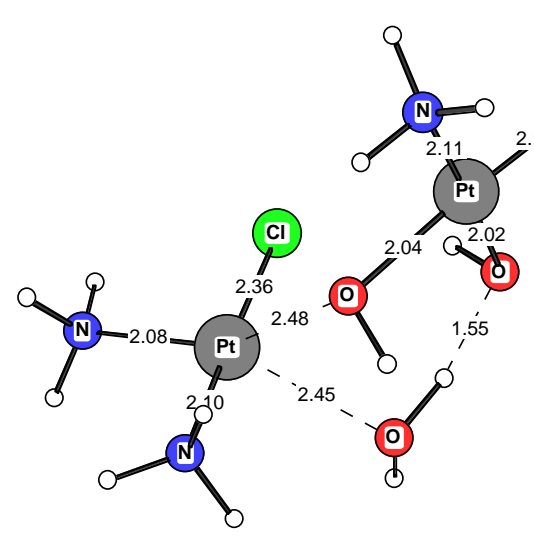

TS BF 4
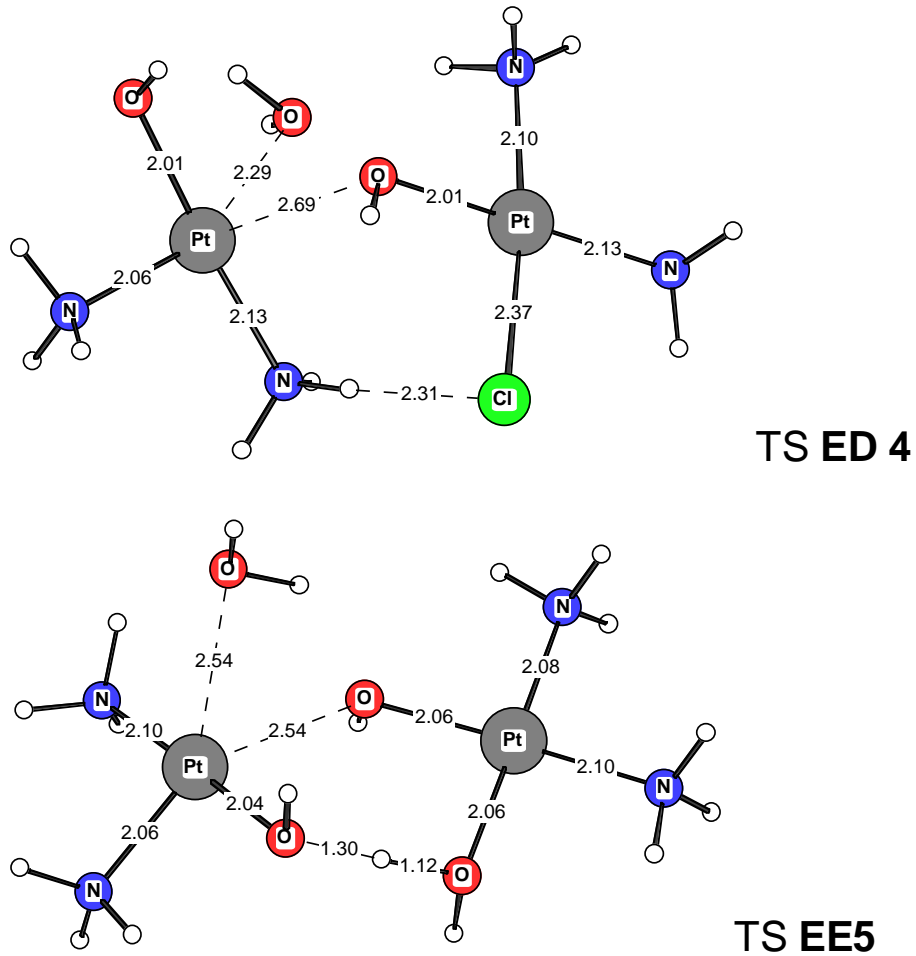

TS EE5 


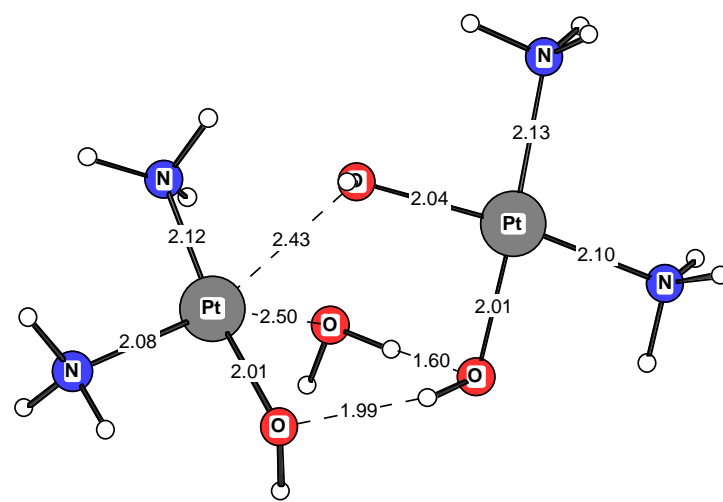

TS EF 6

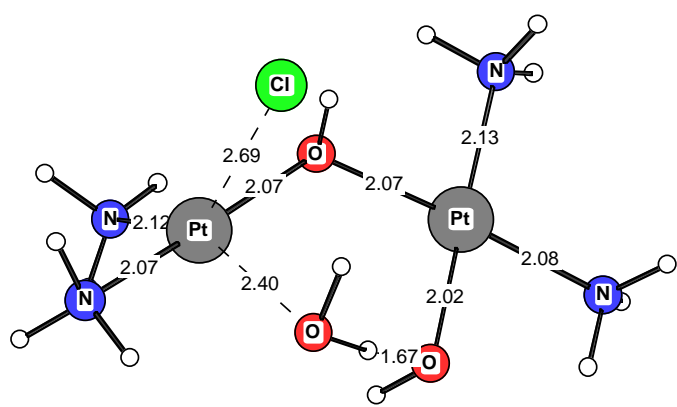

TS 45
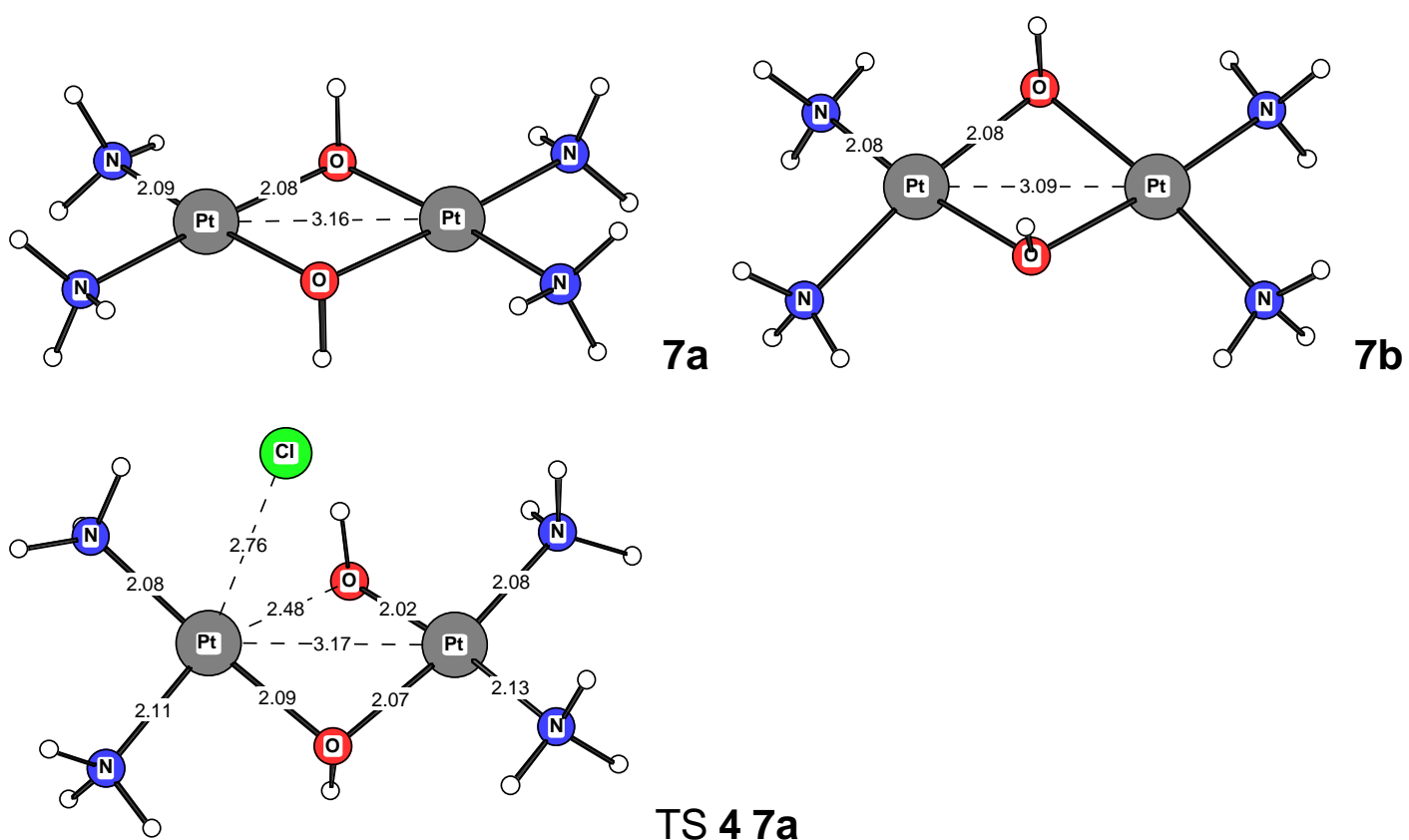

TS 4 7a 

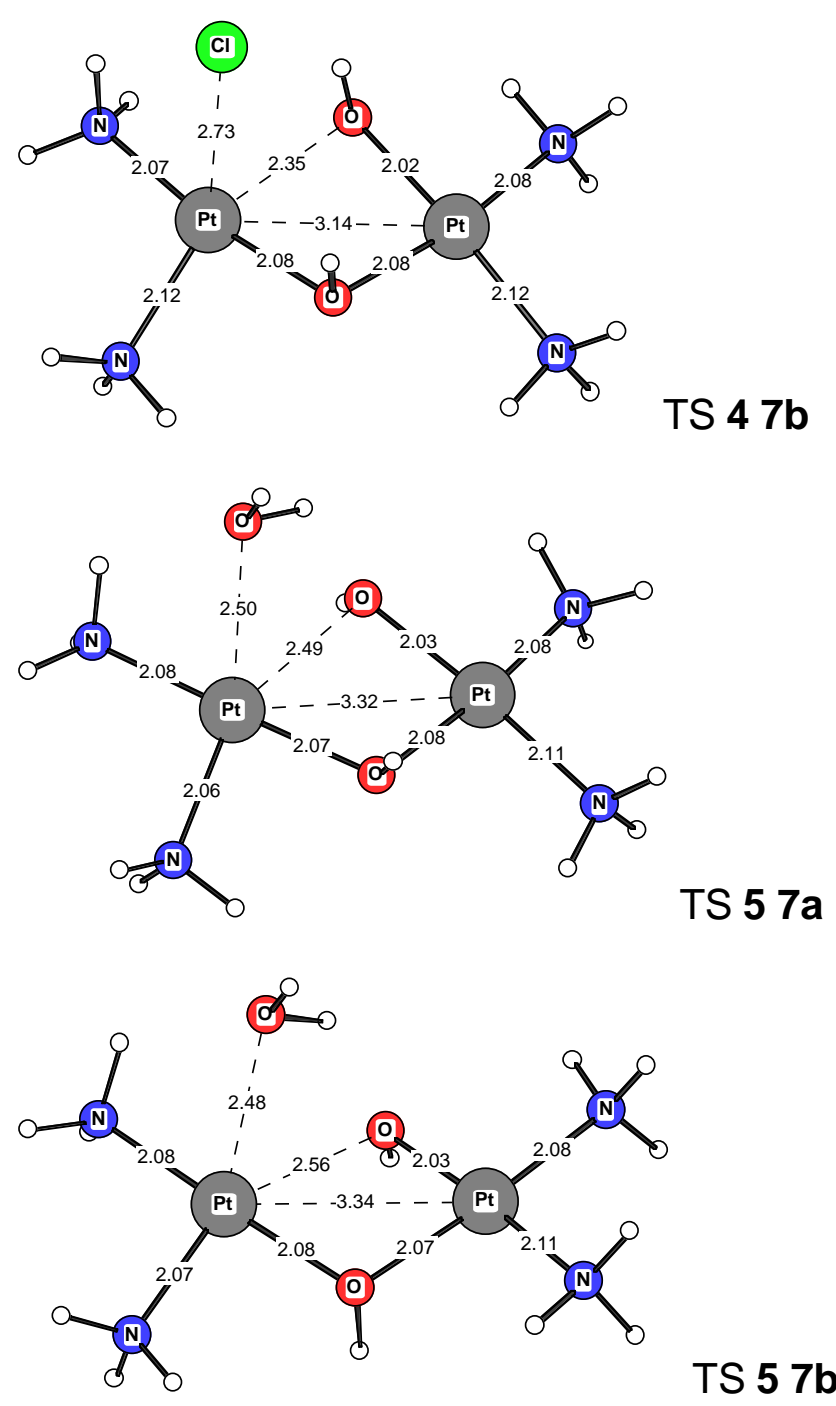

TS 5 7b

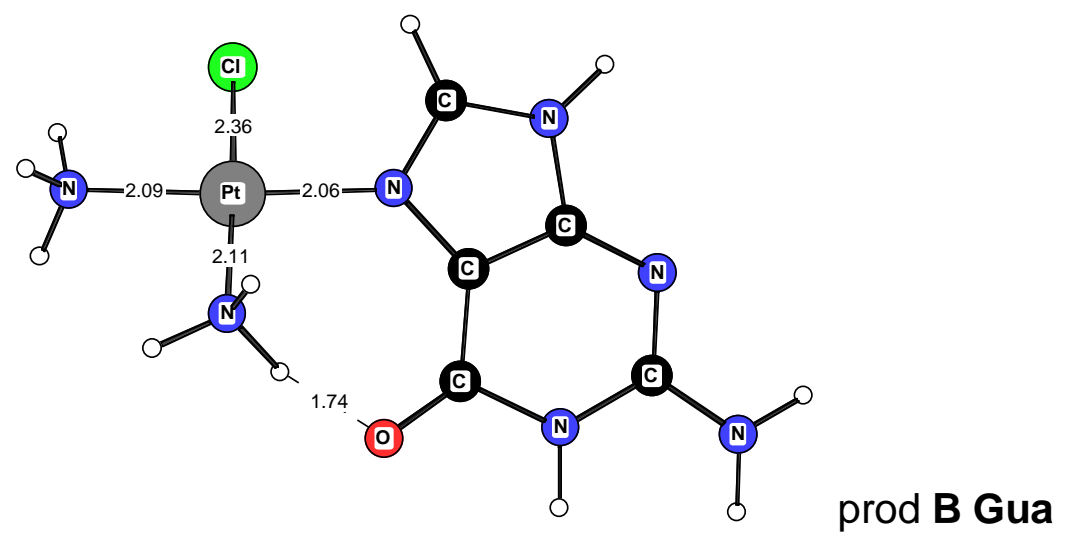



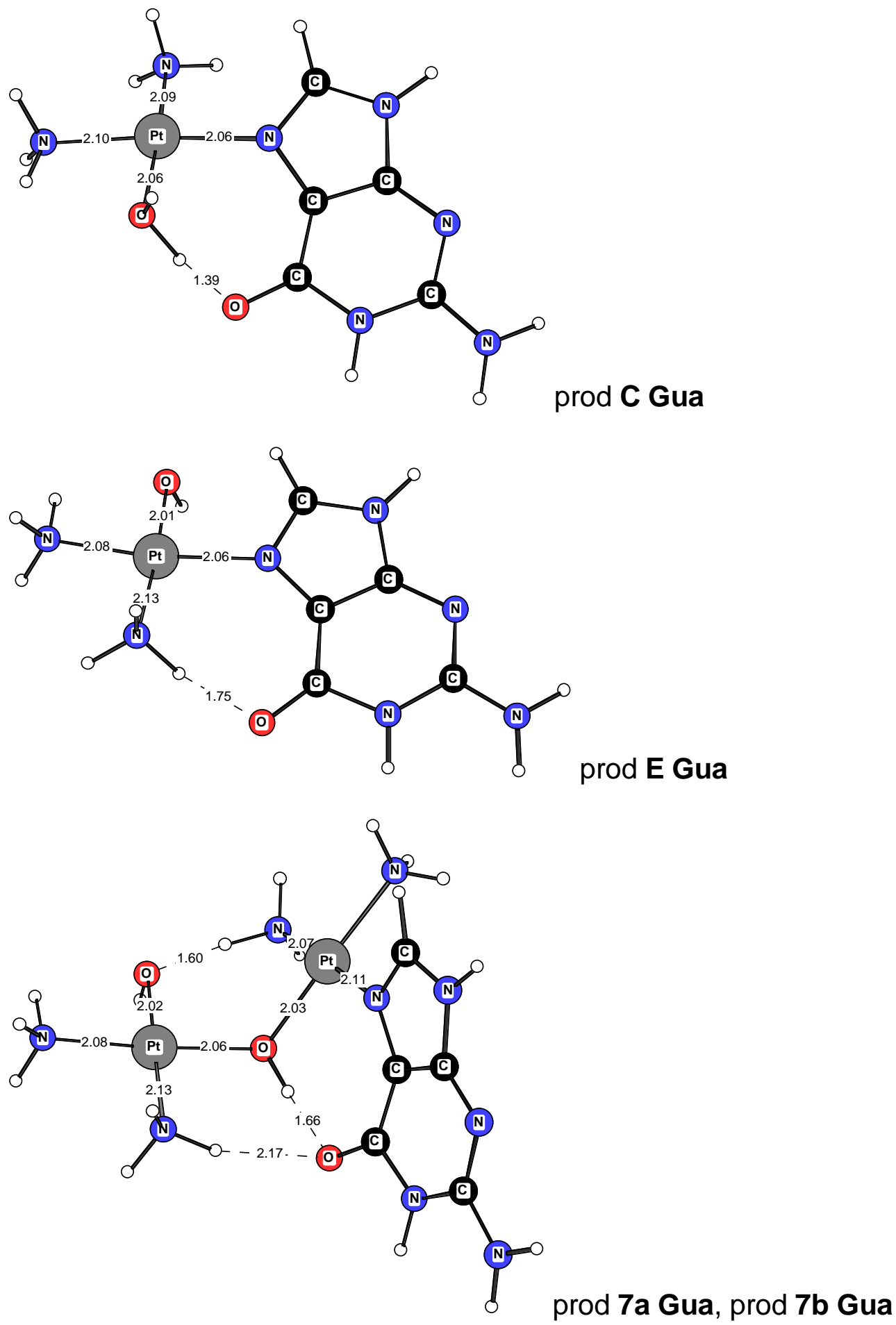

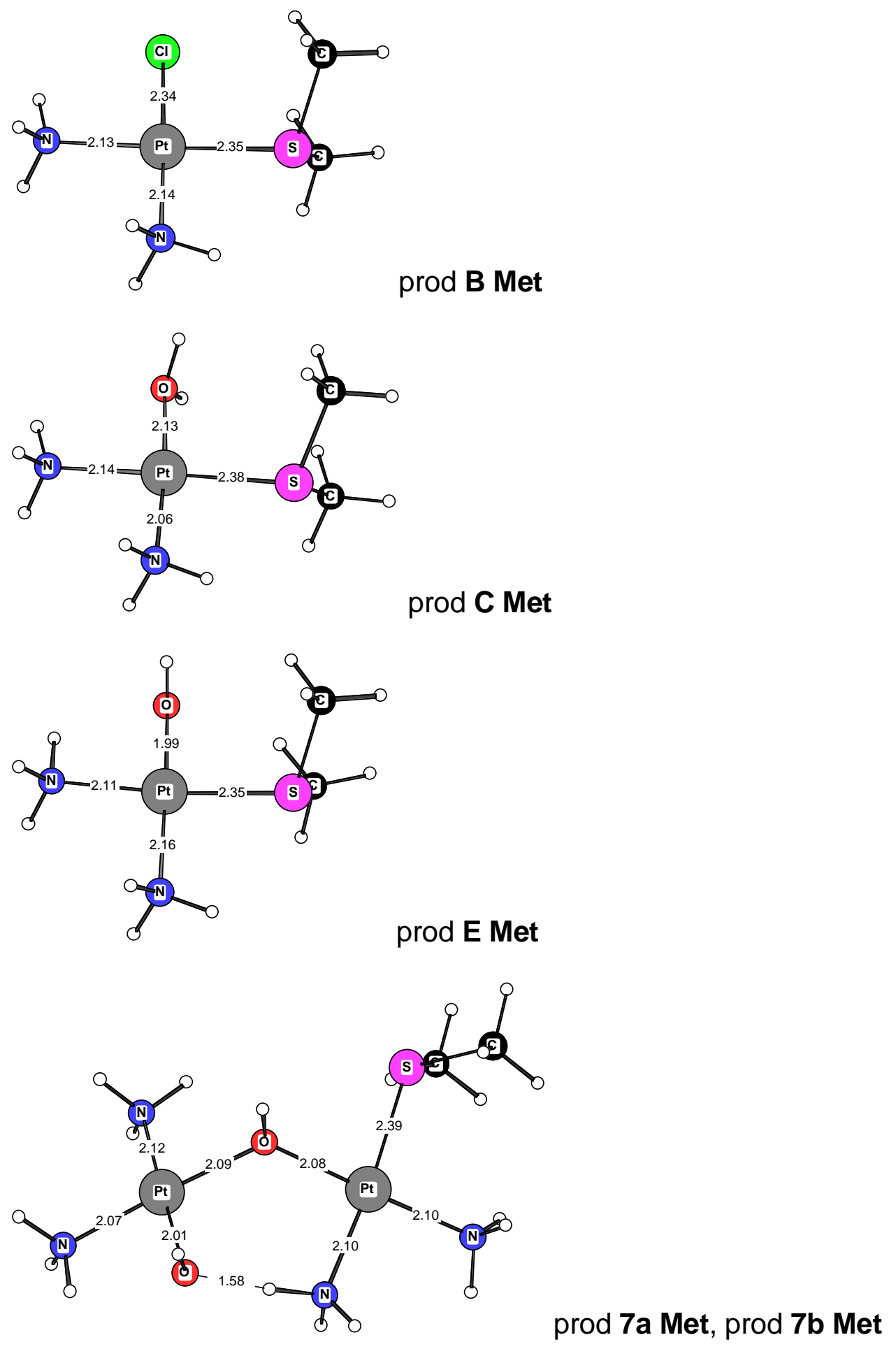

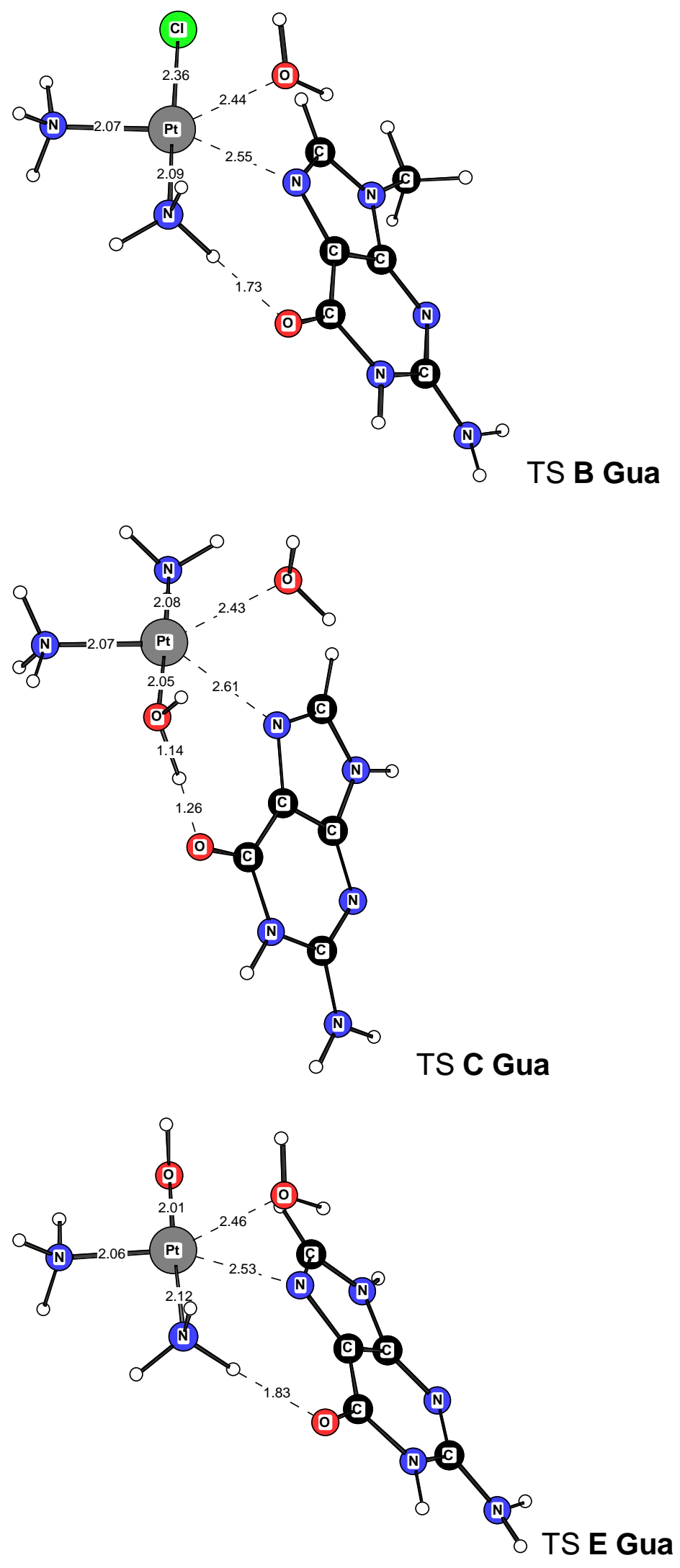


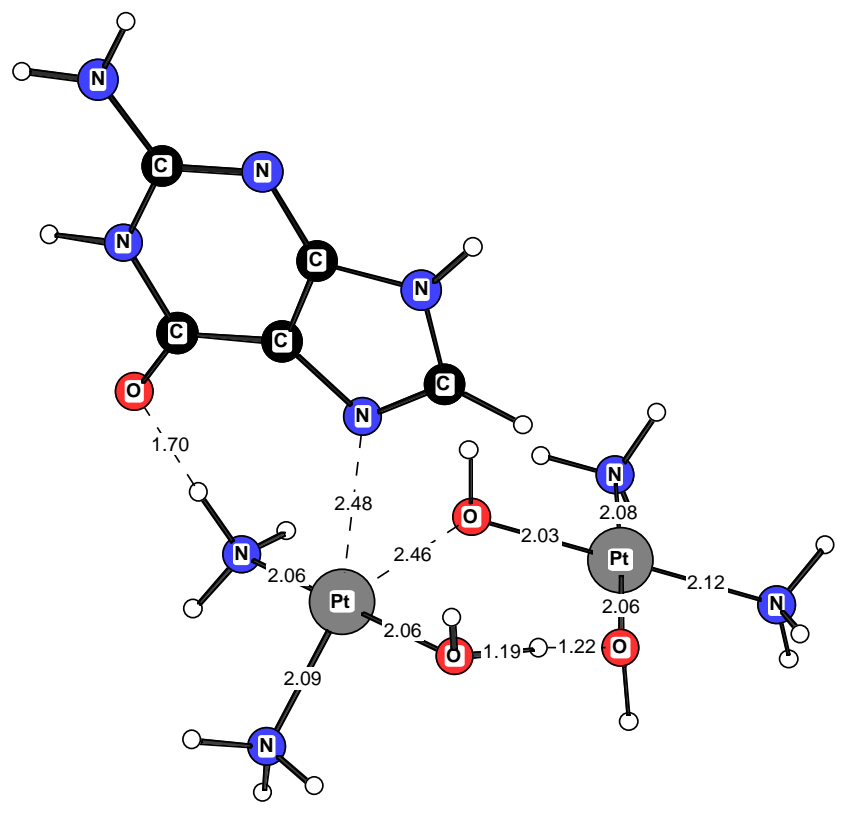

TS 5 Gua

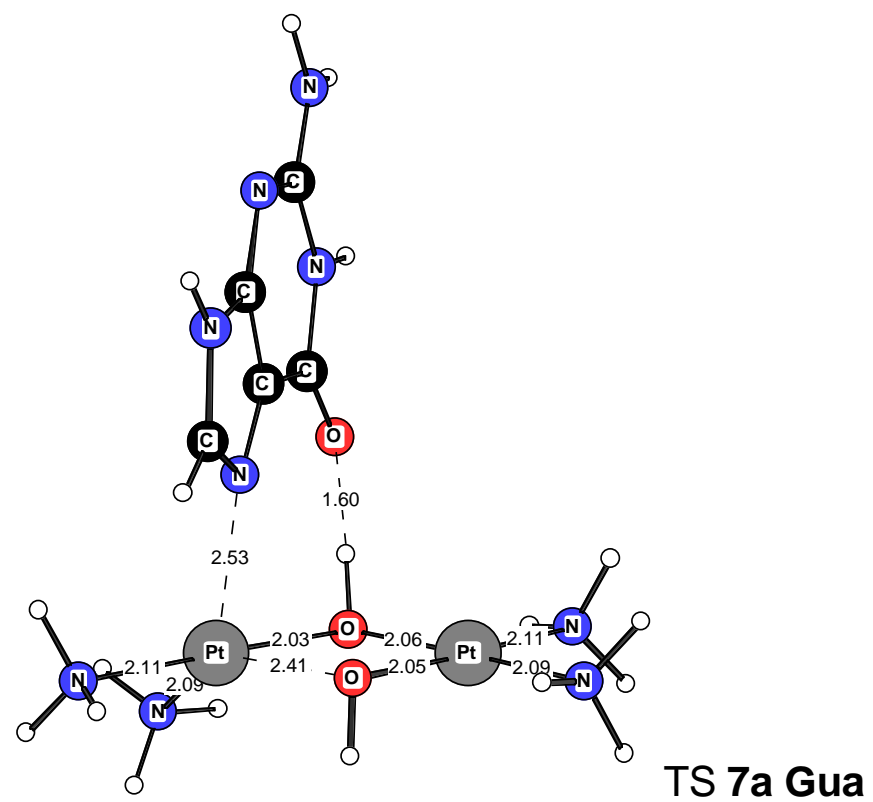



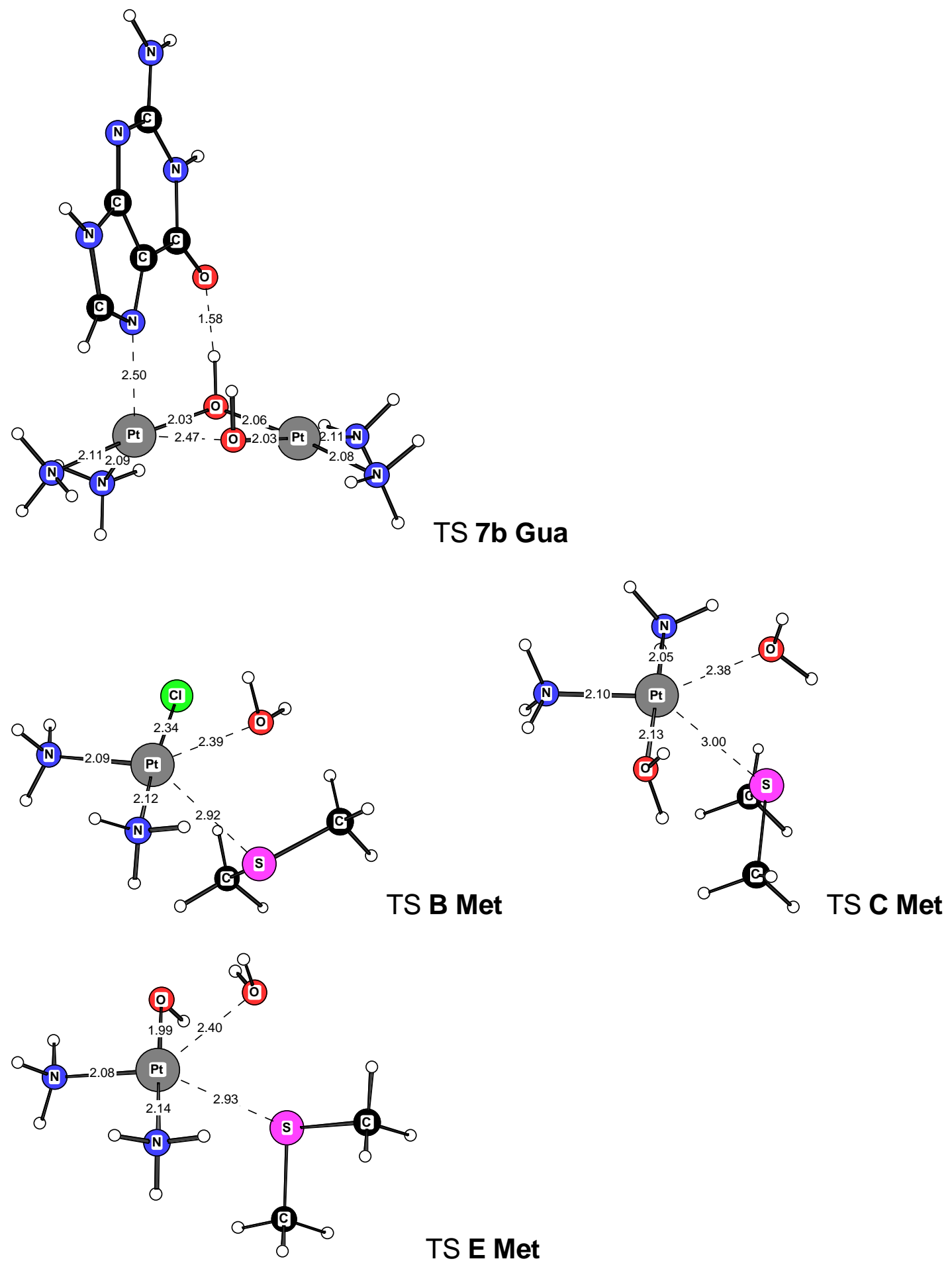

TS E Met 


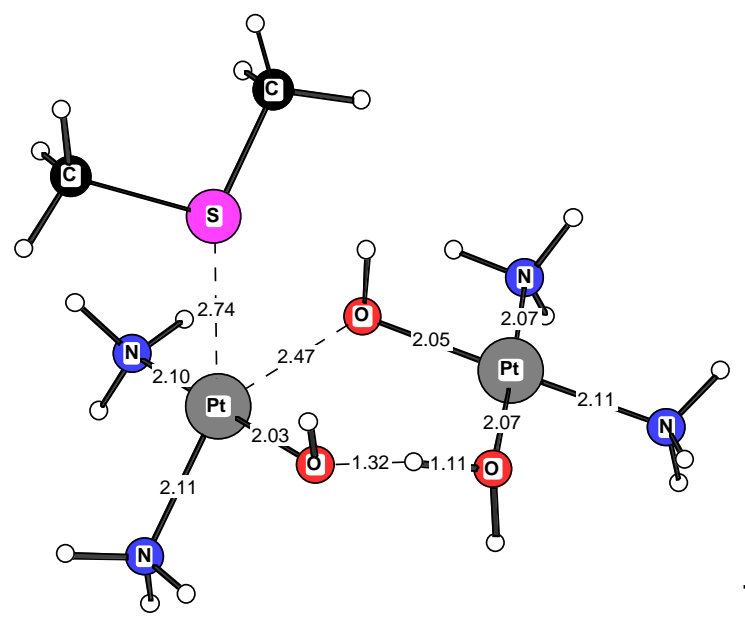

TS 5 Met

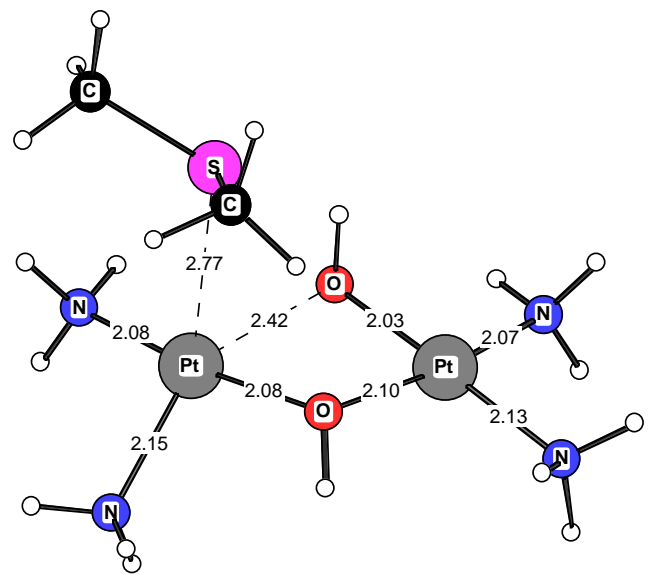

TS 7a Met
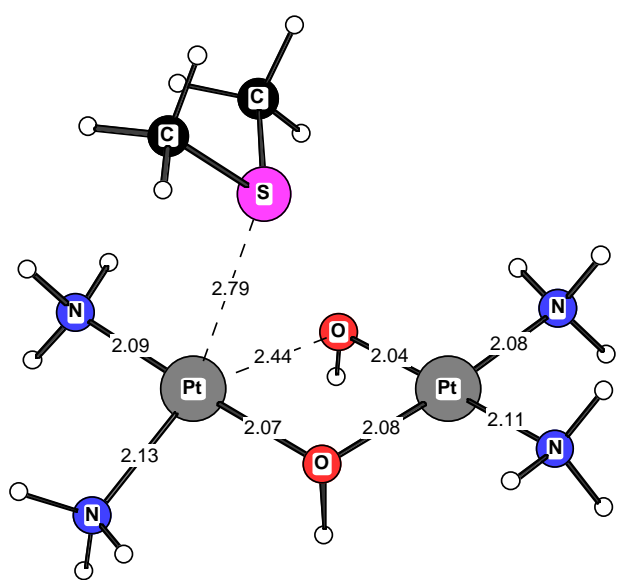

TS 7b Met 


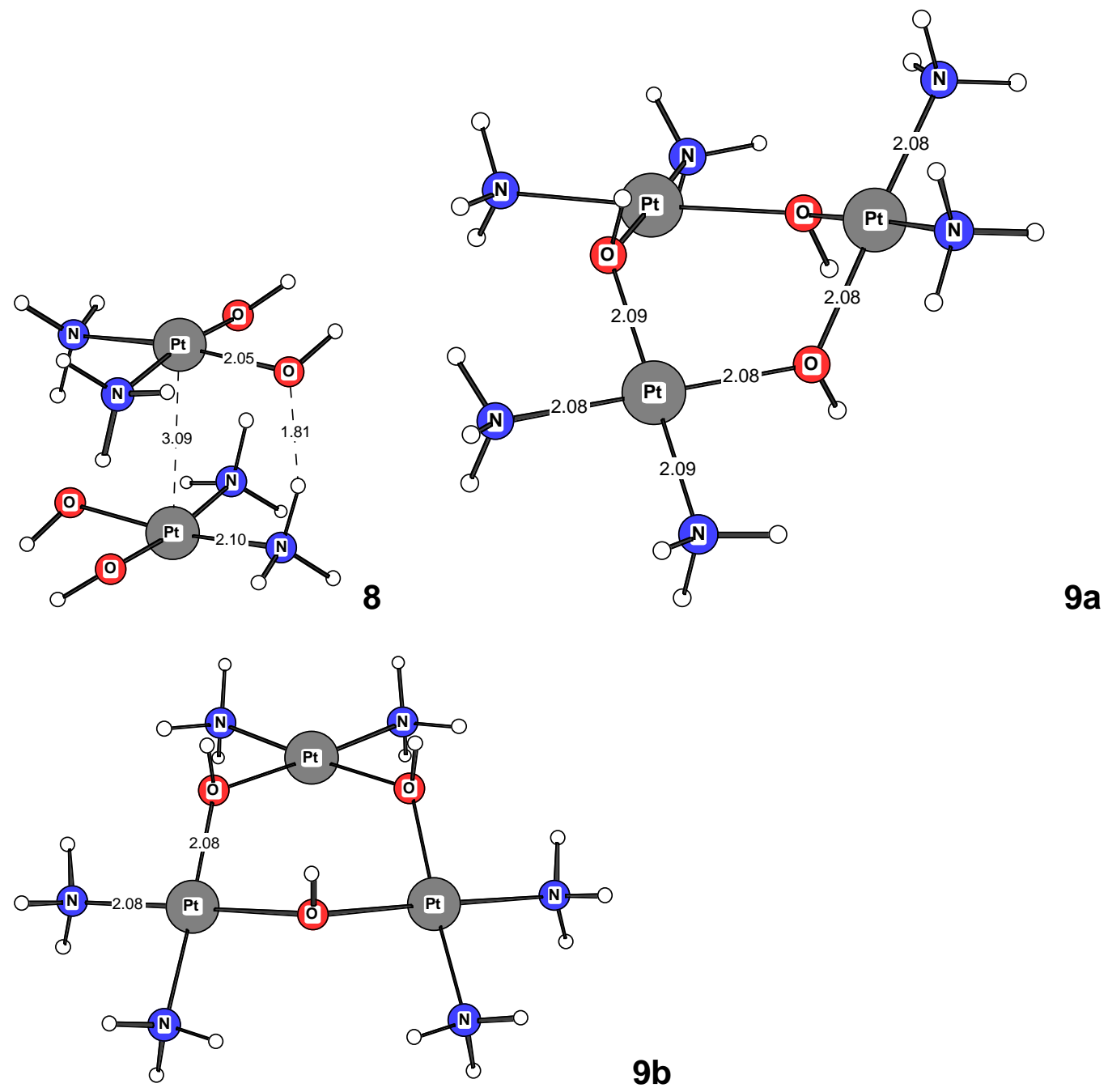

Complete Reference 42. Gaussian 98: Frisch, M. J.; Trucks, G. W.; Schlegel, H. B.; Scuseria, G. E.; Robb, M. A.; Cheeseman, J. R.; Zakrzewski, V. G.; Montgomery, J. A.; Stratmann, R. E.; Burant, J. C.; Dapprich. S.; Milliam, J. M.; Daniels, A. D.; Kudin, K. N.; Strain, M. C.; Farkas, O.; Tomasi, J.; Barone, V.; Cossi, M.; Cammi, R.; Mennucci, B.; Pomelli, C.; Adamo, C.; Clifford, S.; Ochterski, J.; Petersson, G. A.; Ayala, P. Y.; Cui, Q.; Morokuma, K.; Malick, D. K.; Rabuck, A. D.; Raghavachari, K.; Foresman, J. B.; Cioslowski, J.; Ortiz, J. V.; Stefanov, B. B.; Liu, G.; Liashenko, A.; Piskorz, P.; Komaromi, I.; Gomberts, R.; Martin, R. L.; Fox, D. J.; Keith, T. A.; Al-Laham, M. A.; Peng, C. Y.; Nanayakkara, A.; Gonzalez, C.; Challacombe, M.; Gill, P. M. W.; Johnson, B. G.; Chen, W.; Wong, M. W.; Andres, J. L.; Head-Gordon, M.; Replogle, E. S.; Pople, J. A. Gaussian Inc., Pittsburgh, PA, 1998. 